\title{
A Comparison on Selected Physiological Variables between Two Models of Elliptical Cross Trainers 比較雨款饰圆運轉機的生理狀況
}

\author{
Lobo LOUIE Yuen Ting LAM \\ Department of Physical Education, \\ Hong Kong Baptist University, HONG KONG
}

雷雄德 林宛廷

香港浸會大學體育學系

\begin{abstract}
Elliptical cross trainer has become popular for cardio respiratory fitness training. Since new models of elliptical trainers are manufactured for better design and more effective in workout, the present study attempted to compare the physiological variables of the latest model of elliptical trainer, Precor AMT 100i, with the previous model, Precor EFX 576i. A total of 30 female university students participated in the 12-minute incremental elliptical test. Heart rate data and energy expenditure were recorded. Results showed that the latest elliptical model required higher heart rate and energy output, comparatively. However, it appeared that overestimation in energy expenditure was found from the machine-shown data, as compared to the actual metabolic measurement. All data between $60 \%, 80 \%$ heart rate reserve and the equivalent perceived exertion of subjects was found to be no correlation.
\end{abstract}

\section{摘要}

本文探討兩種棈圓運轉機對生理變數的影响。邀請了30位大學女生, 在兩款楉圓運轉機 (Precor EFX576i，Precor AMT 100i) 上進行練習, 收集心跳率、攝氧量、能量消耗及主觀感覺疲勞等數據。結果顯示: 新款型號的樥圓運轉機對身體的生理要 求較大, 而且兩種椭圓運轉機的預測數值, 比實際量度的略大。

\section{Introduction}

Today, people are more aware of their health and fitness. The World Health Organization (WHO) (2007) stated that health is a state of complete physical, mental and social well-being and not merely the absence of disease or infirmity. According to the American College and Sports Medicine (ACSM) and American Heart Association's recommendation (AHA) (2007), regular physical activity is an important behavior for individual and population health. Cardio respiratory fitness is an essential component to the overall health. Cardio respiratory training, which can enhance heart function and contribute to fitness, refers to the ability to perform large muscle, repetitive, moderate-to high-intensity exercise for an extended of time. Resistance and strength training are the examples of cardio respiratory training. In early 1990s, sports enthusiasts started to explore new instrument that not only could practice for cardio respiratory fitness, but also lowered injury rate or even for rehabilitation purpose. Desirably, elliptical cross trainer had been developed as a new aerobic exercise modality with resistance training, and was able to capture the popularity of participants in both fitness clubs and the home consumer market (Egaña, \& Donne, 2004).

Recent research such as Jahn, Borgerpoepping, Nordenskjold, and Dettmer (2007), indicated that elliptical cross trainer is an effective mode of exercise for 
improving aerobic fitness and cardiovascular health. The fitness apparatus were getting more popular among the exercise population. A recent report by the Sporting Goods Manufacturers Association of the United States indicated that the population of elliptical trainer users had grown by $170 \%$ from 2000 to 2005 (Lu, Chien, \& Chen, 2007). Moreover, elliptical trainer was among the top three "most essential" pieces of equipment reported by the respondents answering an open-ended question in the IDEA survey (Keller, 2006).

Elliptical cross trainer is one of the best exercise machines out there in terms of effectiveness, convenience, and user-friendliness. It is not only good for cardio respiratory fitness, but also for musculoskeletal system. The machine is able for practicing forward and backward motion (also called dual action motion) with different resistant levels in order to train for various groups of muscles. "Your leg muscles, buttocks, back, and abdomens do the pedaling work, while you sculpt your biceps, triceps, and several other upper body muscles with a fluid arm swinging motion"(p.4, The Crosstraining Report, 2005). Since large muscle groups of lower extremities are involved to perform elliptical exercise, the cardio respiratory fitness can be trained. Participants can also achieve full body workout by selecting a model with handles or arm poles.

Backward walking may be as good as forward walking especially for women's health. Terblanche, Page, Kroff, and Venter (2004) found that backward walk or run training could improve cardio respiratory fitness and significantly changed in body composition in young women. As stationing at pedals, practicing backward walking on elliptical cross trainer is safe. Jahn et al. (2007) found that exercise on elliptical cross trainer with backward motion created even higher mean heart rates than forward motion on low to moderate intensity exercise.

Gorman (2005) stated that elliptical trainers could minimize the potential injuries from impact and stress on the joints, especially ankles, knees and even hips. Hence, people with joint problems are also able to practice elliptical trainers for both recovery and cardiorespiratory exercise. Practicing on an elliptical machine does not generate large force or moments at the knee joint, although compressive forces increase at higher incline workloads (Knutzen, Lawson, Brilla, \& Chalmers,
2007). Moreover, according to Darryl, Nikolai, Shantanu, and Clifford (2008), less knee joint load was found in patients who practiced on elliptical machines. Gorman (2005) mentioned that elliptical allows people to achieve aerobic goals without worrying about sprains or ligament tears. Researchers recommended that elliptical exercise for rehabilitation should be considered not only the joint loading, but also the muscle strength especially around the knee (Lu et al., 2006).

The Crosstraining Report (2005) stated that a low impact training machines like an elliptical trainer allows workout without aggravating old injuries such as knees, ankles, hips, and back. Also, elliptical trainers mimic running without the foot strike, causes less impact on joints than jogging and running. Women with various injuries, especially knee pain, can use elliptical trainer as a substitute for high-impact activities (Kettles et al., 2006). According to Knutzen, McLaughlin, Lawson, Row, and Martin (2008), mechanics of elliptical stride made a valuable contribution to fitness and rehabilitative arenas. More than that, elliptical cross trainer is also good for pregnant women or the elderly, owing to the low impact nature of the machine. The resistance levels and stride length are easily adjusted to fit individuals in different health level. As a result, elliptical cross trainer suits most of the population with different gender, wide range of age, and even injured people and pregnant women.

Exercising on elliptical trainers would produce similar workout compared to treadmills. Mercer, Dufek, and Bates (2001) indicated that elliptical trainer was comparable to treadmill in training intensity, as the $\mathrm{VO}_{2} \max$ and $\mathrm{HR}$ had been shown to be similar between treadmill running and elliptical trainer exercise. Besides, elliptical cross trainer produced similar maximal values of both male and female during incremental exercise to fatigue compared to treadmill running (Dalleck, Kravitz, \& Robergs, 2004). Furthermore, the elliptical cross trainer was found to be able to offer a variety of intensities that appropriated for most individuals, comparable to treadmill exercise (Mier, $\&$ Feito, 2006). Such data could also be found on the test for patients. Studies designed for coronary artery disease patients also proved the significant higher workout (based on VO2, HR and VE) on elliptical fitness trainer than on treadmill exercise at equivalent levels of RPE (Sweitzer et al., 2002). 
The purpose of the study was to compare the selected physiological variables of university female students when performing on two models of elliptical cross trainers. Heart rate (HR) responses and energy expenditure (EE), together with collection of Rating of Perceived Exertion (RPE) would be monitored and compared while subjects reached to $60 \%$ and $80 \%$ heart rate reserve (HRR).

\section{Method}

\section{Subjects}

Thirty female students aged between 19 and 25 from the Hong Kong Baptist University, were invited to take part in this study. All subjects were free of any cardiopulmonary or respiratory dysfunction. The health status of subjects was ascertained by the Physical Activity Readiness Questionnaire (PAR-Q). Each of the subjects was provided informed written consent prior to the test.

\section{Testing Apparatus}

The apparatus adopted in this study included two models of elliptical cross trainers, a metabolic cart, a heart rate monitor, a facemask with turbine, and a board of Borg's Rating of Perceived (RPE) scale. The two elliptical cross trainers, the Elliptical Fitness Crosstrainer EFX 576i (Precor inc., Woodinville, WA) and the Adaptive Motion Trainer AMT 100i (Precor inc., Woodinville, WA), were utilized for the incremental elliptical test. Gas exchange data was recorded breath-bybreath and analyzed using a metabolic cart (Metamax 3B, Cortex, Germany). The heart rate monitor (S625, Polar Electro, Finland) was used to measure heart rate responses continuously during the test. A board of the Borg's RPE scale, which was measured on a scale of 6 to 20, was used to identify the subjects' exertion. This scale was explained to the subjects prior to the test as if 6 corresponds a resting state and 20 corresponds extremely exhausted that individual was unable to continue.

\section{Procedures}

Subjects were instructed not to engage in strenuous exercise for 24 hours before testing, to refrain from big meal within 2 hours of testing section, and to wear comfortable clothing and sports shoes for testing. A 12-minute incremental elliptical test was designed for the two models of elliptical cross trainers and all subjects performed two tests on two separate days. The testing order on the two elliptical cross trainers was randomly assigned to avoid testing effect. All the incremental elliptical tests were conducted in the Dr. Stephen Hui Research Center for Physical Recreation and Wellness located in the Hong Kong Baptist University, with the temperature and relative humidity at 22 degree Celsius and $70 \%$, respectively. Before the test, subject's body weight, height and BMI were measured by TANITA body composition analyzer (TBF410, Japan) and a wall mounted stadiometer. A heart rate monitor was placed around the subject's chest, and HR data was shown directly when subject performed on the elliptical trainers. The metabolic cart was calibrated before each subjects' test, followed by connecting with turbine properly with a facemask. After linking to the metabolic cart, subjects' energy expenditure (kcal) was displayed continuously throughout the test. The baseline HR of subjects was once recorded for 2 minutes during quiet sitting, followed by the calculation of $60 \%$ and $80 \%$ HRR according to their baseline HR. A minute warm up on the elliptical cross trainer was provided with minimal resistance level. During the warm up session, subjects were provided instruction for the incremental elliptical test. The duration of test was 12 minutes. The resistance was started from level 1 to level 12, with 1-level increased in each minute. The test consisted of forward motion only and subjects performed with arm pole among the test.

\section{Incremental Elliptical Test}

When the test started, subjects' age and weight were entered into the elliptical trainers for the estimation of energy expenditure. HR was recorded each minute from the HR monitor, while EE was marked each 3 minutes from both the elliptical trainers and metabolic cart. For AMT 100i, no incline could be set so that there was no restriction on stride length. It only varied based on the strides of subjects. For EFX 576i, the stride length was set as incline level 10 out of 20 levels. Started from the test, subjects were dictated and reminded to maintain the cadence between 100 and 120 strides per minute throughout the 12-minute test until the end of the test (see Figure 1). 
Figure 1. Protocol of the 12-minute Incremental Elliptical Test.

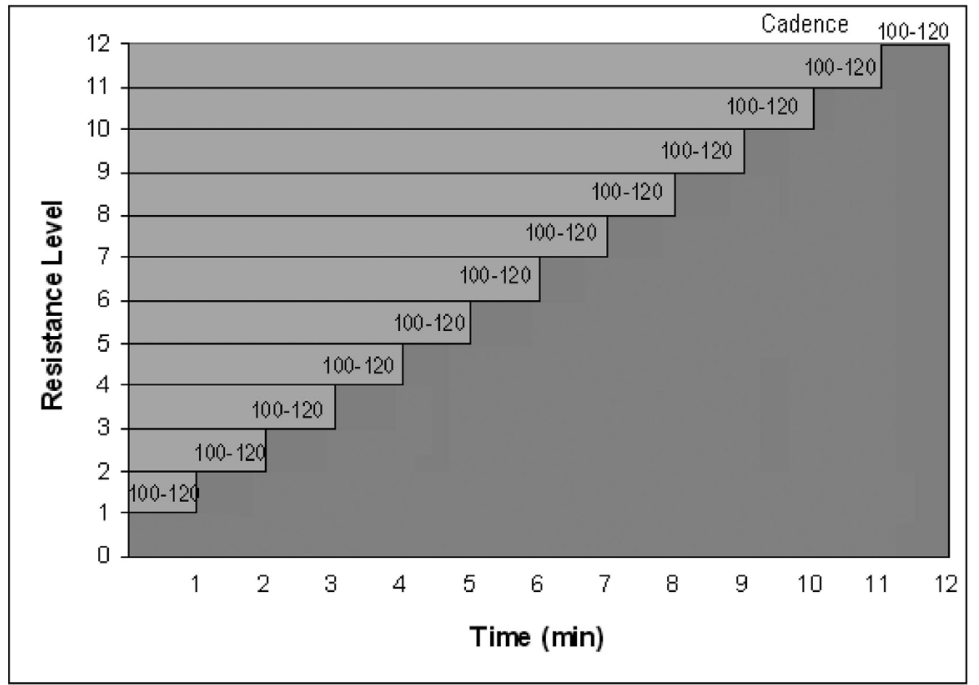

\section{Perceived Exertion}

During the test, subjects were asked for the report on the perceived exertion scale twice. Once the subjects reached their $60 \%$ and $80 \%$ HRR, they were requested to indicate the feeling of exertion. Owing to wearing a facemask, which connected to the metabolic cart, subjects were instructed to point on the board of Borg's RPE scale in order to prevent the difficulty on speaking. In case subjects did not reach the $60 \%$ or $80 \%$ HRR throughout the test, no data of RPE would be collected.

\section{Data Analysis}

Statistical Package for Social Science (SPSS) for window 15.0 version computer program was used for all the statistical calculations. The mean, standard deviation, minimum and maximum values of subjects' age, height, weight, BMI, resting HR, 60\% HRR and $80 \%$ HRR were computed. A 2 x 4 repeated measures ANOVA design was executed to compare energy expenditure of the $3 \mathrm{rd}$, 6th, 9th and 12th minutes throughout the test between the latest and traditional models of elliptical cross trainers. Pearson Product Movement Coefficient of Correlation (r) was used to examine the correlation between \% HRR and RPE. An alpha level of .05 was used for all statistical tests.

\section{Results}

Thirty female university students completed all tests. The physical characteristics of the subjects including age, weight, and height for BMI, resting heart rate for the generation of $60 \%$ and $80 \%$ HRR were summarized in Table 1.

Table 1. Physical Characteristics of the Subjects $(\mathrm{N}=30)$.

\begin{tabular}{llll}
\hline Variables & Minimum & Maximum & Mean \pm SD \\
\hline Age & 19.00 & 25.00 & $21.33 \pm 1.37$ \\
Weight $(\mathrm{kg})$ & 42.60 & 65.70 & $52.00 \pm 6.55$ \\
Height $(\mathrm{cm})$ & 150.00 & 178.00 & $159.33 \pm 5.29$ \\
BMI & 17.30 & 26.70 & $20.45 \pm 2.05$ \\
Resting HR (bpm) & 61.00 & 84.00 & $71.57 \pm 6.23$ \\
$60 \%$ HRR (bpm) & 143.00 & 152.60 & $147.83 \pm 2.42$ \\
$80 \%$ HRR (bpm) & 170.80 & 176.80 & $173.33 \pm 1.42$ \\
\hline
\end{tabular}


The descriptive statistics of the $60 \%$ and $80 \%$ HRR of subjects' equivalent resistance levels were presented in Table 2a and Table 2b. All subjects reached to $60 \%$
HRR while five subjects and eight subjects did not reach to $80 \%$ HRR when performing on AMT 100i and EFX $576 \mathrm{i}$, respectively.

Table 2a. Descriptive Statistics when Subjects Reached 60\% HRR in Corresponding Resistance Level on the Two Elliptical Cross Trainers $(\mathrm{N}=30)$.

\begin{tabular}{lllll}
\hline Elliptical Trainers & \multicolumn{3}{l}{ Resistance } & Level \\
\cline { 2 - 4 } AMT 100i & Mean & Mode & Minimum & Maximum \\
EFX 576i & 4.4 & 2 & 1 & 12 \\
& 7.3 & 9 & 2 & 12 \\
\hline
\end{tabular}

Table 2b. Descriptive Statistics when Subjects Reached 80\% HRR in Corresponding Resistance Level on the Two Elliptical Cross Trainers ( $\mathrm{N}=25$ for AMT100i, N=22 for EFX756i).

\begin{tabular}{lllll}
\hline \multirow{2}{*}{ Trainer Model } & Resistance Level & & \\
\cline { 2 - 5 } AMT 100i & Mean & Mode & Minimum & Maximum \\
EFX 576i & 8.6 & 7 & 3 & 12 \\
\hline
\end{tabular}

Figure 2 illustrated the mean heart rate responses of subjects in each minute throughout the 12-minute incremental elliptical test and the variation of heart rate responses when subjects performed on the two different models of elliptical cross trainers.

Figure 2. Variation of Mean HR Responses from the Two Elliptical Trainers ( $N=30)$.

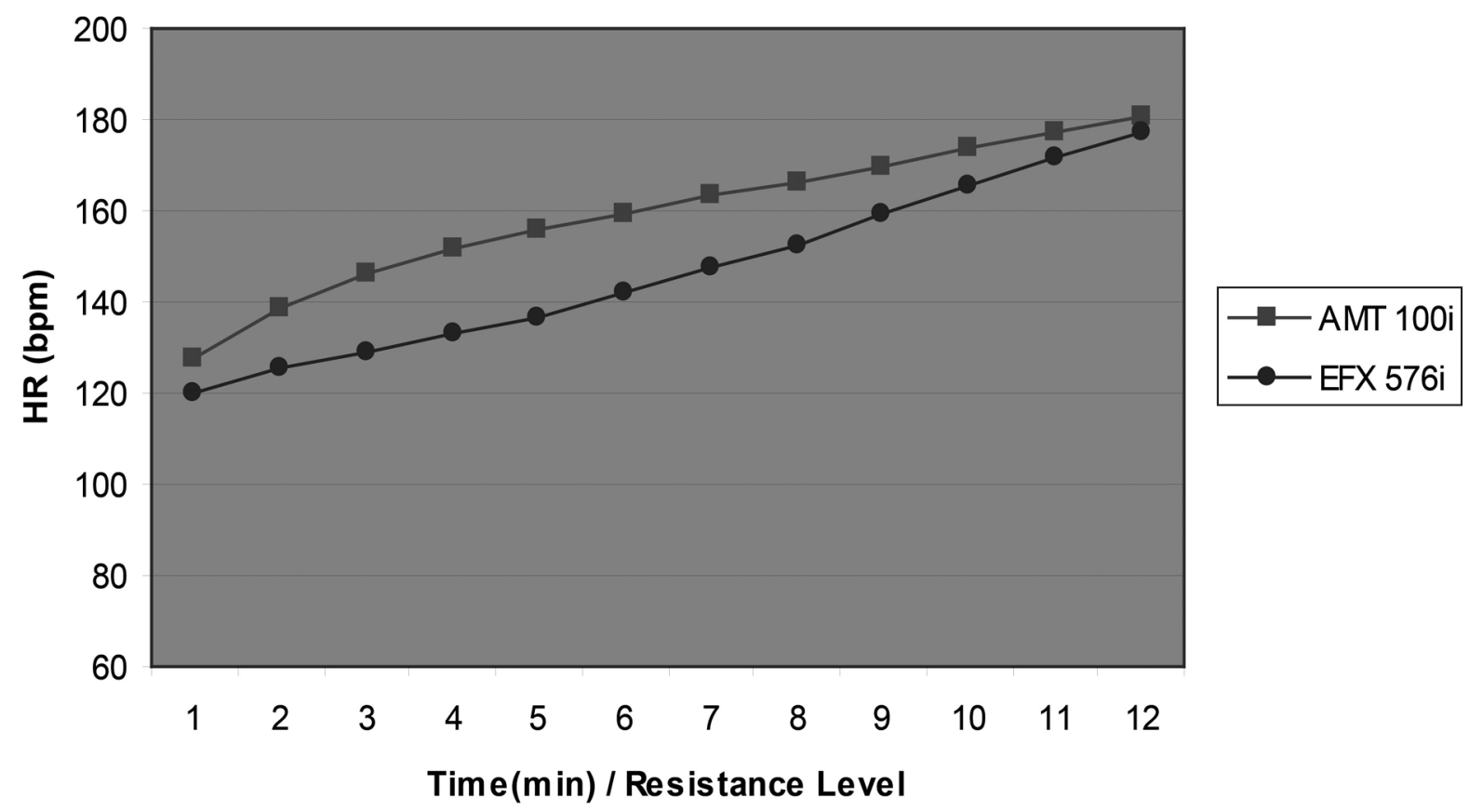


Table 3 showed the descriptive statistics including mean, standard deviation and mean difference of subjects' energy expenditure (kcal) accumulated in every 3-minute throughout the test. EE was collected from both elliptical trainers and metabolic cart when subjects performed on the AMT 100i and EFX 576i. The results of mean EE were illustrated in graph pattern in Figure 3. As there was distinct overestimation of EE from elliptical trainers, the percentage of overestimation was shown in Table 4.

Table 3. Descriptive Statistics of Subjects' EE by Estimation and Actual Measurement ( $N=30$ ).

\begin{tabular}{|c|c|c|c|c|}
\hline \multicolumn{5}{|c|}{ Energy Expenditure (kcal/min) } \\
\hline & & Mean \pm SD & & \\
\hline Resistance Level & Collected from: & AMT $100 \mathrm{i}$ & EFX $576 \mathrm{i}$ & Mean Difference \\
\hline \multirow[t]{2}{*}{3} & $\mathrm{E}$ & $24.07 \pm 2.40$ & $17.40 \pm 1.16$ & 6.67 \\
\hline & A & $16.03 \pm 2.90$ & $13.94 \pm 1.91$ & 2.09 \\
\hline \multirow[t]{2}{*}{6} & $\mathrm{E}$ & $50.97 \pm 4.63$ & $38.67 \pm 2.35$ & 12.31 \\
\hline & A & $35.43 \pm 6.39$ & $30.05 \pm 3.66$ & 5.37 \\
\hline \multirow[t]{2}{*}{9} & $\mathrm{E}$ & $79.27 \pm 6.80$ & $63.50 \pm 3.57$ & 15.76 \\
\hline & A & $57.95 \pm 9.26$ & $48.69 \pm 5.74$ & 9.26 \\
\hline \multirow[t]{2}{*}{12} & $\mathrm{E}$ & $109.23 \pm 9.15$ & $91.38 \pm 4.92$ & 17.85 \\
\hline & A & $82.33 \pm 12.50$ & $70.67 \pm 8.06$ & 11.66 \\
\hline
\end{tabular}

E: Estimation from elliptical trainers

A: Actual measurement from metabolic cart

Figure 3. An Overall Result of Subjects' Mean EE by Estimation and Actual Measurement ( $\mathrm{N}=30)$.

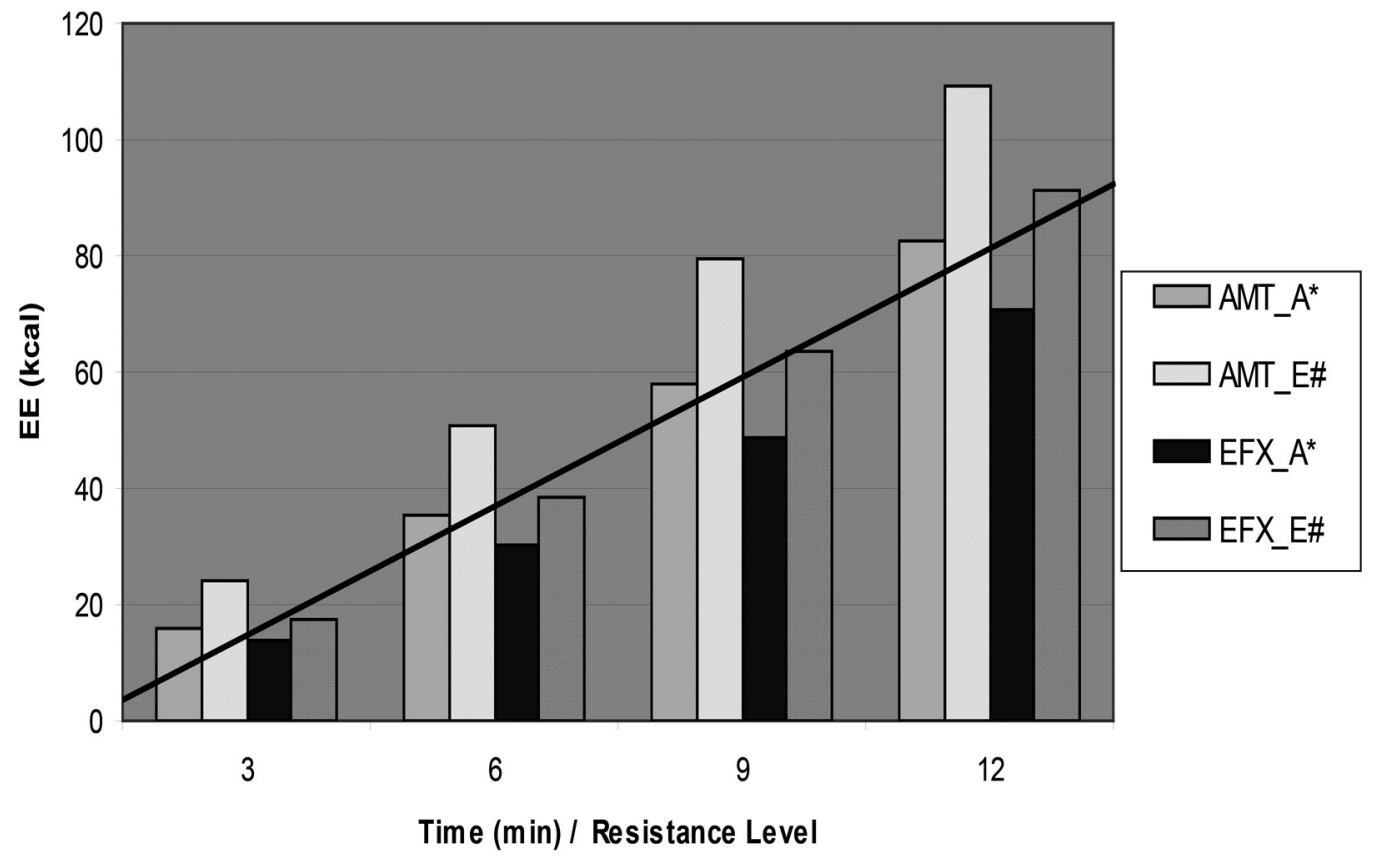

A*: Actual measured EE from metabolic cart

E\#: Estimated EE from elliptical trainers 
Table 4. Overestimation of Mean EE on AMT 100i and EFX 576i in Four Levels ( $N=30$ ).

\begin{tabular}{lll}
\hline Level & $\%$ of Overestimation & \\
\cline { 2 - 3 } 3 & AMT 100i & EFX 576i \\
6 & 50.16 & 24.82 \\
9 & 43.86 & 28.69 \\
12 & 36.79 & 30.42 \\
\hline
\end{tabular}

A 2 x 4 repeated measures ANOVA design was utilized to compare four different stages of energy expenditure during the 12-minute incremental elliptical test (measurement made at the 3rd, 6th, 9th and 12th minutes) within two models of elliptical trainers, AMT 100i and EFX 576i. Two sets of data were collected from metabolic cart as the actual measurement of EE (Table 5a) and the elliptical trainers as estimation of EE (Table 5b). Figure 4 and 5 showed the profile plots of estimated marginal means of EE by actual measurement and estimation, respectively.

Table 5a. Results of 2x4 Repeated Measures ANOVA Designed for Two Models of Elliptical Trainers and EE, Actual Measurement from Metabolic Cart.

\begin{tabular}{|c|c|c|}
\hline \multicolumn{3}{|l|}{ Multivariate Tests (b) } \\
\hline Effect & $\mathrm{F}$ & Sig. \\
\hline EE(actual) & 1005.241(a) & $.000 *$ \\
\hline EE(actual)* Model & 9.049(a) & $.000 *$ \\
\hline
\end{tabular}

a Exact statistic

b Design: intercept+ Model

Within Subjects Design: EE(actual)

$* \mathrm{p}<0.05$

Table 5b. Results of 2x4 Repeated Measures ANOVA Designed for Two Models of Elliptical Trainers and EE, Estimation from Elliptical Trainers.

\begin{tabular}{|c|c|c|}
\hline \multicolumn{3}{|l|}{ Multivariate Tests (b) } \\
\hline Effect & $\mathrm{F}$ & Sig. \\
\hline EE(estimate) & 3986.607(a) & $.000^{*}$ \\
\hline EE(estimate)* Model & 730.36(a) & $.000 *$ \\
\hline
\end{tabular}

a Exact statistic

b Design: intercept+ Model

Within Subjects Design: EE(estimate)

$* \mathrm{p}<0.05$ 
Figure 4. Profile Plot of Estimated Marginal Means of EE from Metabolic Cart (Actual measured).

\section{Estimated Marginal Means of EE (actual measured)}

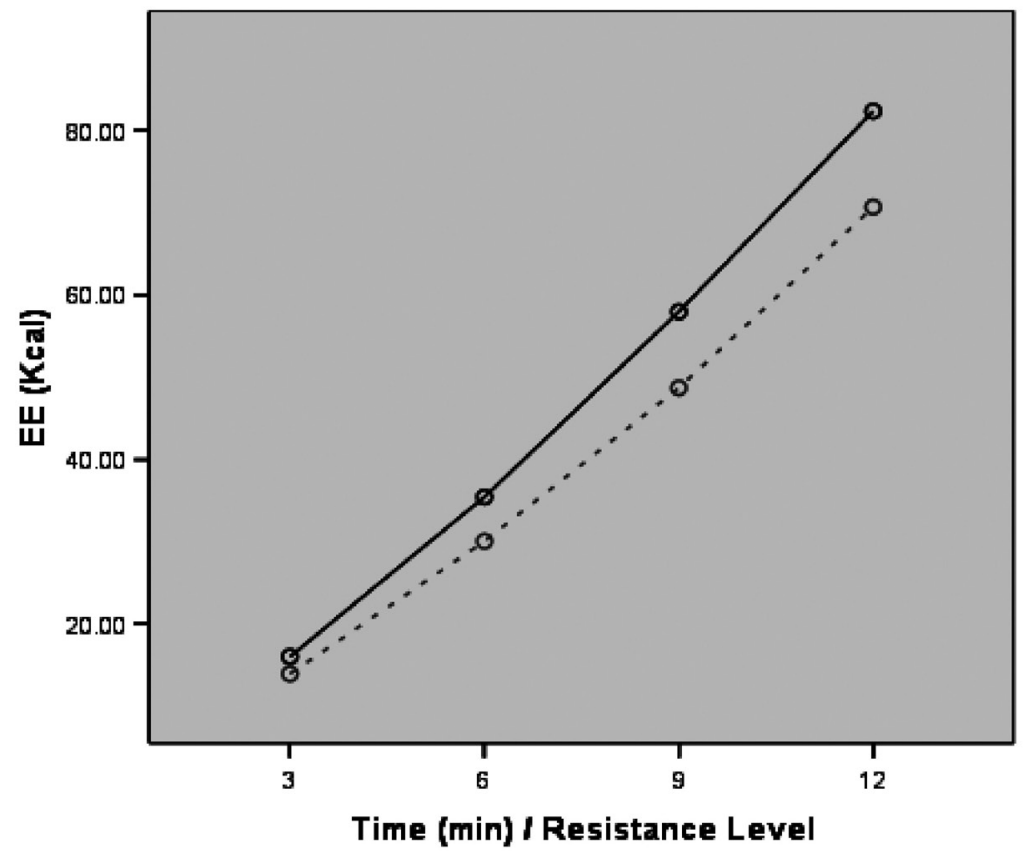

Machine

- AMT 100i

- - EFX 576i

Figure 5. Profile Plot of Estimated Marginal Means of EE from Elliptical Trainers (Estimated).

\section{Estimated Marginal Means of EE (estimated)}

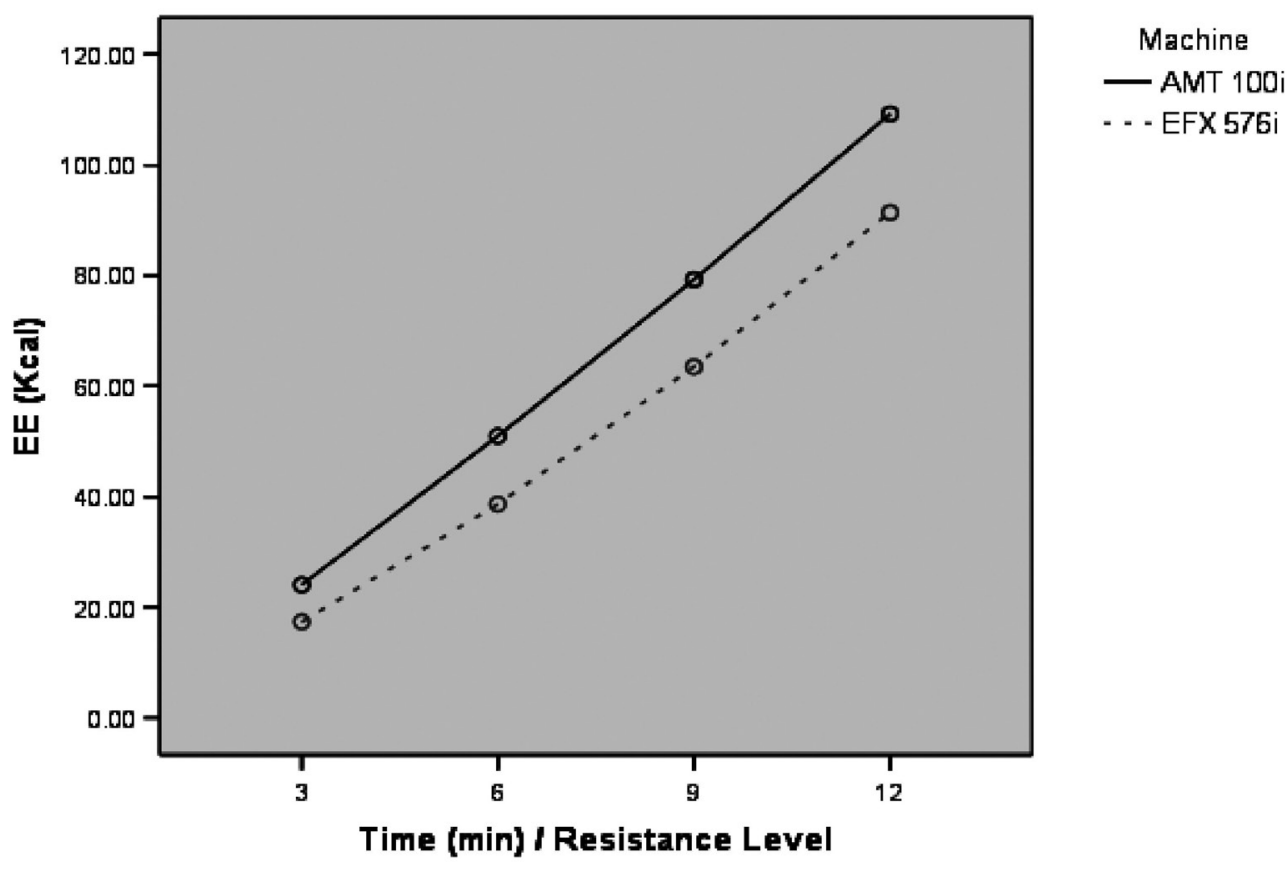


The relationship between subjects' HRR and equivalent perceived exertion when they reached $60 \%$ and $80 \%$ HRR was analyzed by the Pearson's correlation test. Table $6 \mathrm{a}$ and Table $6 \mathrm{~b}$ showed the results of $60 \%$ and
$80 \%$ HRR, respectively. Since five subjects did not reach $80 \%$ HRR on AMT 100i as well as eight subjects did not reach $80 \%$ HRR on EFX 576i, RPE would not be collected from these subjects.

Table 6a. Pearson's Correlation Test between 60\% HRR and Equivalent RPE in Two Elliptical Cross Trainers $(\mathbf{N}=30)$.

\begin{tabular}{llll}
\hline Trainer Model & $\mathrm{r}$ & $\mathrm{r} 2$ & $\mathrm{p}$ \\
\hline AMT 100i & .070 & .005 & .357 \\
EFX 576i & -.027 & .001 & .444 \\
\hline
\end{tabular}

Table 6b. Pearson's Correlation Test between 80\% HRR and Equivalent RPE in Two Elliptical Cross Trainers ( $\mathrm{N}=25$ for AMT100i, N=22 for EFX756i).

\begin{tabular}{lccc}
\hline Trainer Model & $\mathrm{r}$ & $\mathrm{r} 2$ & $\mathrm{p}$ \\
\hline AMT 100i & .208 & .043 & .159 \\
EFX 576i & .097 & .009 & .333 \\
\hline
\end{tabular}

The finding displayed that there was no significant relationship between \% HRR and RPE on AMT 100i $(60 \%$ HRR, r $=0.070 ; 80 \%$ HRR, r=0.208, $>>0.05)$ and EFX 576i $(60 \%$ HRR, r=-0.027; 80\% HRR, r=0.097, $\mathrm{p}>0.05$ ). Table 7a and $7 \mathrm{~b}$ showed the relationship between RPE and the corresponding resistance level when subjected reached $60 \%$ and $80 \%$ HRR, analyzed by the Pearson's correlation test.

Table 7a. Pearson's Correlation Test between RPE and Subjects' Corresponding Resistance Level when Reached 60\% HRR in Two Elliptical Cross Trainers ( $N=30)$.

\begin{tabular}{llll}
\hline Trainer Model & $\mathrm{r}$ & $\mathrm{r} 2$ & $\mathrm{p}$ \\
\hline AMT 100i & $.548^{* *}$ & .300 & .001 \\
EFX 576i & .319 & .102 & .043 \\
\hline
\end{tabular}

** Correlation is significant at the 0.01 level (1-tailed)

Table 7b. Pearson's Correlation Test between RPE and Subjects' Corresponding Resistance Level when Reached 80\% HRR in Two Elliptical Cross Trainers ( $=25$ for AMT100i, N=22 for EFX756i).

\begin{tabular}{llll}
\hline Trainer Model & $\mathrm{r}$ & $\mathrm{r} 2$ & $\mathrm{p}$ \\
\hline AMT 100i & $.705^{* *}$ & .497 & .000 \\
EFX 576i & .006 & .000 & .489 \\
\hline
\end{tabular}

** Correlation is significant at the 0.01 level (1-tailed) 


\section{Discussions}

Exercising on the latest model, AMT 100i, appeared to have higher HR responses than performing on the traditional model, EFX 675i. The increasing rate of HR of AMT 100i was a bit higher for the first five minutes than EFX 576i, while EFX 576i only showed a gradual and steady rise throughout the test. Descriptive statistics presented the mean resistance level when subjects reached $60 \%$ HRR on both elliptical cross trainers. It was obvious that lower resistance level was needed to reach $60 \%$ HRR when exercising on AMT 100i (mean= level 4.4) than on EFX 576i (mean= level 7.3). Similar results were reported when subjects reached $80 \%$ HRR during the incremental elliptical test on AMT 100i (mean= level 8.6) and EFX 576i (mean= level 10.4). Furthermore, five tested females could not reach $80 \%$ HRR on AMT 100i whilst eight of them could not reach the same \% HRR on EFX 576i. Most subjects reached 60\% HRR at resistance level 2 and 9 on AMT 100i and EFX 576i, respectively, and they mostly reached $80 \% \mathrm{HRR}$ at resistance level 7 and 12 on AMT 100i and EFX 675i, respectively. These results revealed that subjects exercising on EFX 576i might require higher resistance level to gain the similar HR when compared to AMT 100i. In other words, the dual-plane design of the latest model of elliptical cross trainers can generally lead to higher workout.

The latest model of elliptical cross trainer was believed to provide superior machine design with better workout when compared with the previous elliptical trainer. Referring to the collected data on the four-stage energy expenditure (measured at the 3rd, 6th, 9th and 12th minute) of AMT 100i and EFX 576i, subjects exercising on AMT 100i generally spent more EE than on EFX 576i. The more time and the greater resistance level, the higher the EE. Gradual linear rise on mean value of $\mathrm{EE}$ from the 3 rd to 12 th minute was found. In Figure 4 and 5, the profile plots separately presented the significant difference on AMT 100i and EFX 576i. For the actual measured of EE in Figure 4, mean value of EE on AMT 100i appeared slightly higher than EFX 576i at level 3, and it gradually made greater different especially at level 6 and till the end at level 12. The significant difference on EE between AMT 100i and EFX 576i proved that the new model in the same brand Precor, provided a better workout in the same resistance and duration of test. However, a factor influencing the workout of the current study might be the stride length. According to Dolny, Hughes, Caylor and Browder (2004), increasing stride length was an effective method to enhance the cardio respiratory demand during elliptical trainer exercise. Due to the different stride length on AMT 100i not restricted) and EFX 576i (restricted to incline level 10), it was inferred that subjects performing on AMT 100i might put more effort on stride length than on EFX 576i, so as to produce a greater mean values of $\mathrm{EE}$ on the latest elliptical cross trainers.

Using the $2 \times 4$ repeated measures ANOVA approach, collected data from both metabolic cart and elliptical trainers was significantly different on energy expenditure on AMT 100i and EFX 576i. For the actual measurement from metabolic cart in Table 5a, interaction between EE and two models of elliptical trainers was significant $(\mathrm{F}=9.049, \mathrm{p}<0.05)$. As well as the machineestimated $\mathrm{EE}$ in Table 5b, between factors of $\mathrm{EE}$ and two models of elliptical trainers was also significant $(\mathrm{F}=$ 730.36, $\mathrm{p}<0.05)$. The distinct overestimation by elliptical trainers was observed. The percentage of overestimation in all level of test and on both elliptical trainers was found especially in level 3 on AMT 100i (50.16\%). Nevertheless, such results were common in previous studies. Schorner, Terracciano, Hickner, and McCammon (2004) indicated that the caloric expenditure predicted by the elliptical trainer, Precor EFX 546, was 33.4\% $7.7 \%$ higher than the data from metabolic cart. Overestimation of caloric expenditure was found on all tested subjects. Oja and Wilcow (2004) also reported that the machine-reported caloric expenditure was consistently $26 \%$ higher than which generated by calorimetry in different incline levels. The reason of being overestimated might firstly due to the input of personal data into machine. Only age and weight were required to enter for estimation of energy output. However, height which contributed to body surface area was also an important factor influencing the EE during exercise. Therefore, the output of energy estimation was limited. Secondly, the physical characteristic of subjects might differ from the original Precor inc. Subjects in the current research were all Chinese female, while subjects of the original study were mostly westerners. 


\section{Conclusion}

Based on the present findings, higher heart rate response is required when exercising on the latest model of elliptical cross trainer, AMT 100i. Secondly, there is significant difference in energy expenditure between the latest and previous models of elliptical cross trainers. More energy is spent for performing on AMT 100i rather than the traditional EFX 576i. Thirdly, actual measured and estimated of energy expenditure is significantly different. Overestimation in energy output from the machine is found from each levels of the test. Fourthly, there is no relationship between $60 \%, 80 \%$ HRR of subjects and the equivalent rate of perceived exertion. In conclusion, the latest elliptical trainer provides a better workout, contributing to cardio respiratory fitness. Further studies might be conducted utilizing a variety of subjects, such as pregnant women, elderly and people with disabilities.

\section{References}

American College of Sports Medicine. (2007). ACSM's resources for the personal trainer (2nd ed.). Baltimore: Lippincott Williams \& Wilkins.

American College of Sports Medicine, \& American Heart Association. (2007). Physical activity and public health: updated recommendation for adults from the American College of Sports Medicine and American Heart Association. Circulation, 116, 1081-1093.

Batté, A. L., Darling, J., Evans, J., Lance, L. M., Olson, E. I., \& Pincivero, D. M. (2003). Physiological response to a prescribed rating of perceived exertion on an elliptical fitness cross-trainer. Journal of Sports Medicine and Physical Fitness, 43(3), 300-205.

Borg, G. (1998). Borg's perceived exertion and pain scales. Champaign, IL: Human Kinetics.

Cedillo, T. (n.d.). The Precor elliptical - The finest in smooth fat burning workouts. ABC Article Directory. Retrieved February 12, 2009, from http://www. abcarticledirectory.com/Article/The-Precor-Elliptical--The-Finest-In-Smooth-Fat-Burning-Workouts/160455
Corbin, C. B., Welk, G. J., Corbin, R. W., \& Welk, K. A. (2006). Fundamental concepts of fitness and wellness (2nd ed.). New York: The McGraw-Hill.

Dalleck, L. C., Kravitz L., \& Robergs, R. A. (2004). Maximal exercise testing using the elliptical crosstrainer and treadmill. Journal of Exercise Physiology online, 7(3), 94-101.

Darryl, D. D., Nikolai, S., Shantanu, P., \& Clifford, W. C. (2008). The mark coventry award in vivo knee forces during recreation and exercise after knee arthroplasty. Clinical Orthopaedics and Related Research, 446(11), 2605-2611.

Dolny, D., Hughes, N. J., Caylor, R., \& Browder, K. (2004). Effect of varying stride length on cardiorespratory response during elliptical trainer exercise. Medicine \& Science in Sports \& Exercise (Supplement), 36(5), S250.

Egaña, B., \& Donne, B. (2004). Physiological changes following a 12 week gym based stair-climbing, elliptical trainer and treadmill running program in females. The Journal of Sports Medicine and Physical Fitness, 44(2), 141-146.

Gorman, T. (2005). Low risk of injuries from elliptical machines. Ezine Articles. Retrieved April 21, 2009, from http://ezinearticles.com/?Low-Risk-of-Injuries-fromElliptical-Machines\&id=90535

Heyward, V. H. (2006). Advanced fitness assessment \& exercise prescription (5th ed.). Champaign, IL: Human Kinetics.

Howley, E. T. (2001). Type of activity: resistance, aerobic and leisure versus occupational physical activity. Medicine \& Science in Sports \& Exercise, 33(6), S364-S369.

Jahn, N., Borgerpoepping, M., Nordenskjold, A., \& Nettmer, J. (2007). Measurement of metabolic responses on an elliptical cross-trainer: forward vs. backward at Low and moderate intensities. Journal of Undergraduate Kinesiology Research, 2(2), 25-31.

Keller, J. (2006). Don't forget the job description. IDEA Fitness Journal, 3(1). 
Kettles, M., Cole, C. L., \& Wright, B. S. (2006). Women's health and fitness guide. Champaign, IL: Human Kinetics.

Knutzen, K. M., Lawson, A., Brilla, L., \& Chalmers, G. (2007). Knee joint loads during exercise on the elliptical trainers. Medicine \& Science in Sports \& Exercise (Supplement), 39(5), S154-155.

Knutzen, K. M., McLaughlin, W., Lawson, A., Row, B., \& Martin, L. (2008). Ramp position influences lower extremity biomechanics while exercising on the elliptical trainer. Medicine \& Science in Sports \& Exercise (Supplement), 40(5), S340.

Kulig, K., Brener, N. D., Brener., \& McManus, T. (2003). Sexual activity and substance use Among Adolescents by category of physical activity plus team sports participation. Archives of Pediatrics \& Adolescent Medicine. 157, 905-912.

Leung, R. W., Leung, M. L., Chung, P. K., \& Binh, Q. (2002). Validity and reliability of a Chinese-translated perceived exertion scale for children: The children's effort rating table (CERT). The Hong Kong Journal of Sports Medicine and Sports, 15.

Lu, T. W., Chien, H. L., \& Chen, H. L. (2007). Joint loading in the lower extremities during elliptical exercise. Medicine \& Science in Sports \& Exercise, 39(9), 1651-1658.

Mercer, J. A., Dufek, J. S., \& Bates, B. T. (2001). Analysis of peak oxygen consumption and heart rate during elliptical and treadmill exercise. Journal of Sports Rehabilitation, 10(1), 48-56.

Mier C. M., \& Feito, Y. (2006). Metabolic cost of stride rate, resistance, and combined use of arms and legs on the elliptical trainer. Research Quarterly for Exercise and Sport, 77(4), 507-513.

Norman, J. F., Kracl, J., Parker, D., \& Richter, A. (2002). Comparison of the counting talk test and heart rate reserve methods for estimating exercise intensity in healthy young adults. Journal of Exercise Physiology online, $5(1), 15-22$.
Oja, K. M., \& Wilcow, A. R. (2004). A comparison of machine-predicted and measured energy expenditure during elliptical exercise across ramp inclines. Medicine \& Science in Sports \& Exercise (Supplement), 36(5), S247.

Porcari, J., Foster, C., \& Schneider, P. (2000). Exercise response to elliptical trainers. Fitness Management, 16(9), 50-53.

Schorner, M. J., Terracciano, D. C., Hickner, R. C., \& McCammon, M. R. (2004). The PreCor EFX546 elliptical trainer over predicts energy expenditure. Medicine \& Science in Sports \& Exercise, 36(5) Supplement, S249-S250.

Smith, W. (2009). Contemporary nutrition (7th e.d.). New York: McGraw-Hill.

Sweitzer, M. L., Kravitz, L., Weingart, H. M., Dalleck, L. C., Chitwood, L. F., \& Dahl, E. (2002). The cardiopulmonary responses of elliptical crosstraining versus treadmill walking in CAD patients. Journal of Exercise Physiology online, 5(4), 11-15.

Terblache, E., Page, C., Kroff, J., \& Venter, R. E. (2004). The effect of backward locomotion training on the body composition and cardiorespiratory fitness of young women. International Journal of Sports and Medicine, 25, 1-6.

The crosstraining report. (2005). Running \& FitNews, 23(6), 4-5.

World Health Organization. (2007). Mental health: strengthening mental health promotion [Fact Sheet $\left.N^{\circ} 220\right]$. Retrieved from http://www.who.int/mediacentre/ factsheets/fs220/en/

\section{Correspondence:}
Dr. Lobo Louie
Department of Physical Education, Hong Kong Baptist University, Kowloon Tong, Hong Kong. Email: s62591@hkbu.edu.hk 
Appendix A. The latest model of elliptical cross trainer, Precor AMT 100i.

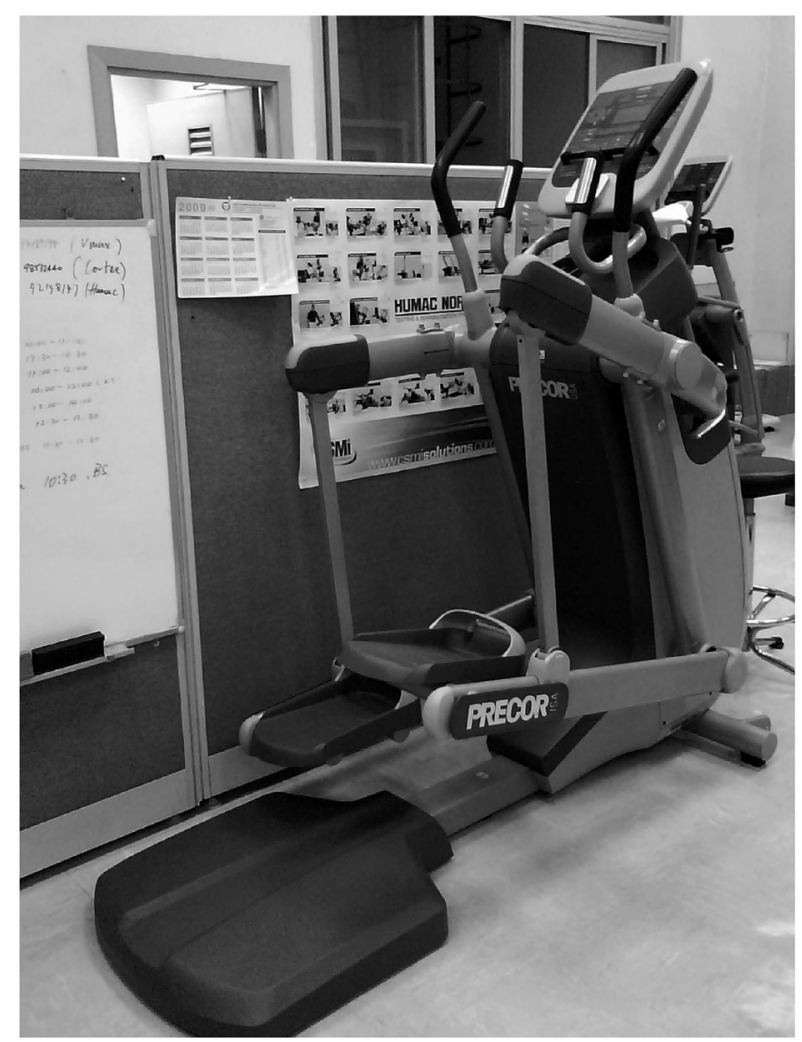

Appendix B. The traditional model of elliptical cross trainer, Precor EFX 576i.

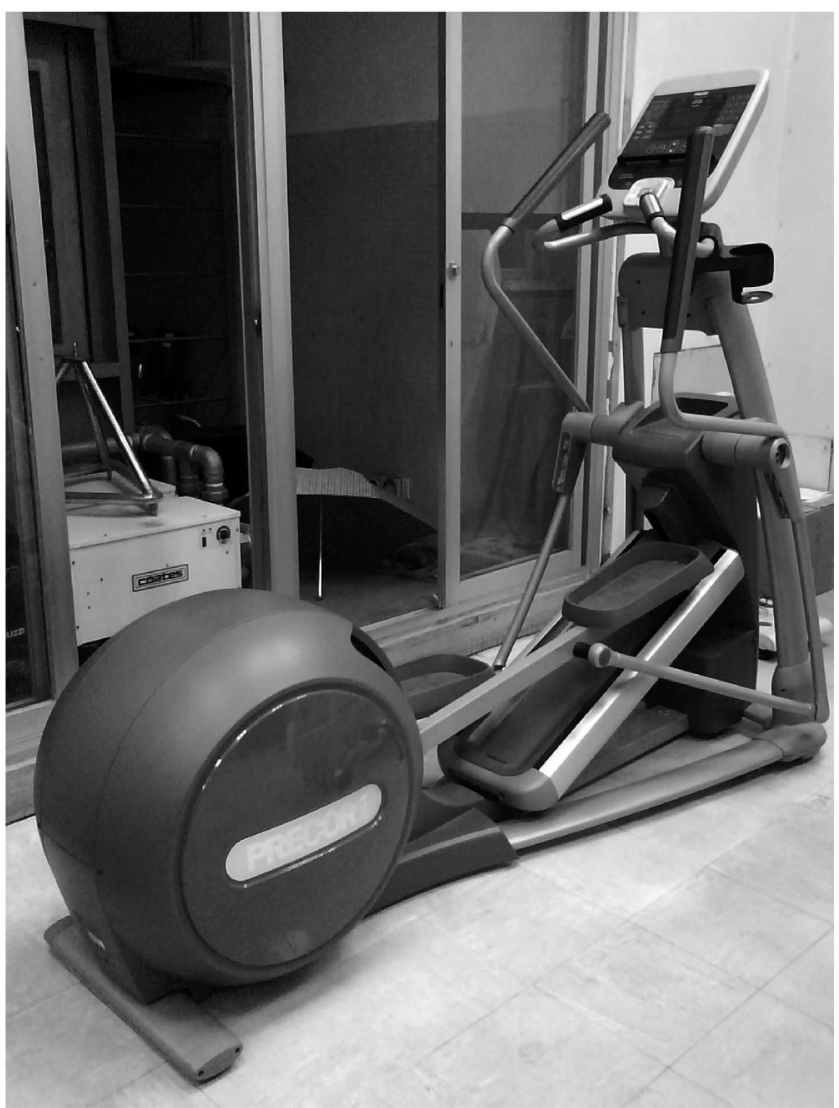

